Trauma Berufskrankh $2014 \cdot$ 16[Suppl 4]:439-443 DOI 10.1007/s10039-014-2086-2

Online publiziert: 17. September 2014

(c) Springer-Verlag Berlin Heidelberg 2014
U.-J. Gerlach · C. Grimme · R. Schoop · M. Borree

Abteilung für Septische Unfallchirurgie und Orthopädie,

Berufsgenossenschaftliches Unfallkrankenhaus Hamburg

\title{
Gelenkinfekt - eine Entität für die spezielle septische Chirurgie
}

\section{Hintergrund}

Eine Gelenkinfektion ist definiert als Befall eines Gelenks durch pathogene Erreger, meist Bakterien, und eine sich anschließende Entzündung. Pilzbedingte Gelenkinfektionen sind nur bei immungeschwächten Personen bekannt.

Eine Infektion eines Gelenks führt unbehandelt zu dessen Zerstörung.

Für die Entstehung einer Gelenkinfektion sind exogene und endogene Ursachen zu unterscheiden [7]. Die endogenen Faktoren stellen laut Literatur [19] die häufigste Ursache für eine Gelenkinfektion dar. In der überwiegenden Zahl der Fälle handelt es sich um eine hämatogene Streuung im Rahmen einer Bakteriämie. Die Infektion kommt dann meist im Rahmen einer Kombination von prädisponierenden Faktoren und der Bakteriämie zustande.

Unter unseren Patienten überwogen mit 95\% der Fälle die exogene Faktoren, in diesem Kontext als gelenknahe Frakturen, v. a. gelenknahe offene Frakturen, oder perforierende Verletzungen. Häufiger entwickeln sich Gelenkinfektionen jedoch nach diagnostischen Arthroskopien und arthroskopischen Operationen (Inzidenz 0,04-0,42\%; [2, 6, 20]), Gelenkpunktionen, intra- oder paraartikulären Injektionen (Inzidenz 0,003-0,42\%, [1, 7, 10, 12]) oder nach Arthrotomien (Inzidenz $\leq 1 \%,[1,8,16])$. Seltener sind sie durch paraartikulär fortgeleitete Infekte, $z$. B. eine verschleppte Bursitis, bedingt.
Nach Eintritt eines bakteriellen Erregers in den Gelenkbinnenraum kommt es zu einer intraartikulären Erregervermehrung und zur Anreicherung von Stoffwechselprodukten bzw. Toxin. Nach $24 \mathrm{~h}$ werden lysosomale Enzyme freigesetzt, nach 48 h zeigen sich klinische Symptome des Infektbeginns. Im Verlauf kommt es zur Freisetzung von Glukosaminen und Leukozytenproteinasen [11]. Nach 5 Tagen beginnt die Zerstörung des Knorpels, welche nach 10 Tagen irreversibel ist. Bereits nach 3 Tagen kommt es zu einer Synoviahypertrophie, nach 11 Tagen zur Pannusbildung. Am 17. Tag nach Infektbeginn ist ein Kapseldurchbruch festzustellen. Untersuchungen ergaben, dass die Arthroserate bereits am 3. Tag nach Beginn der Gelenkinfektion einen deutlichen Anstieg zeigt.

\section{Diagnostik}

Die Diagnosestellung eines akuten Gelenkinfekts ist oft einfacher als die eines chronischen Gelenkinfekts.

Die akute Gelenkinfektion verläuft klinisch wesentlich eindrucksvoller, die bekannten klinischen Entzündungszeichen („rubor“, „calor“, „dolor“ und „functio laesa“; - Abb. 1) sind häufig eindrucksvoll. Die schmerzhaft aufgehobene Beweglichkeit des betreffenden Gelenks in Verbindung mit erhöhten Temperaturen ist typisch für die akute Gelenkinfektion.

Bei der chronischen, schon lange Zeit bestehender Gelenkinfektion können die klinischen Entzündungszeichen fehlen oder nur deutlich abgeschwächt vorhanden sein (• Abb. 2).

Für die Diagnostik wichtig ist eine genaue Anamneseerhebung mit Befragung nach vorausgegangenen, auch längere Zeit zurückliegenden Gelenkpunktionen oder intraartikulären Injektionen oder Arthroskopien. Selbstverständlich muss auch nach Grunderkrankungen, wie u. a. Diabetes mellitus, PCP (primär chronische Polyarthritis), mögliche gelenkferne Streuherde (Tonsillitis, Otitis, Harnwegsinfekt), oder immunsuppressiven Medikamenten gefragt werden.

Die Laborparameter [Leukozyten/ CRP (C-reaktives Protein)/BSG (Blutsenkungsgeschwindigkeit), [17]] zeigen nur beim Frühinfekt eine deutliche Erhöhung. Bei chronischen Gelenkinfektionen sind sie wenig aussagekräftig und haben allenfalls hinweisenden Charakter.

Weiterer wichtiger Baustein in der Diagnostik ist die Gelenkpunktion. Die Inspektion des aspirierten Punktats lässt differenzialdiagnostische Überlegungen zu. Es kann serös, serös-flockig, trübe bis eitrig sein. Ein Teil des Gelenkpunktats sollte zur bakteriologischen Untersuchung in ein mikrobiologisches Labor verschickt, ein weiterer Teil unter GramFärbung im Mikroskop untersucht werden. Dabei muss die Leukozytenzahl im Punktat überprüft werden, ggf. ist auch ein Keimnachweis erforderlich. Bei einer Leukozytenzahl im Punktat $\geq 25.000 / \mu \mathrm{l}$ $\left(\geq 2,5 \times 10^{10} / 1\right.$; [5]) ist von einem Infekt- 


\section{Septische Problemfälle}

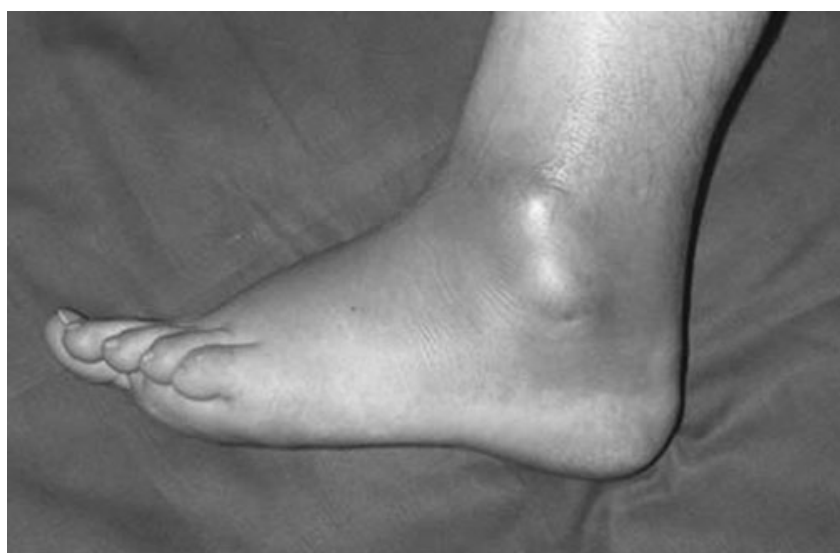

Abb. 1 A Klinisches Bild eines akuten Empyems des oberen Sprunggelenks

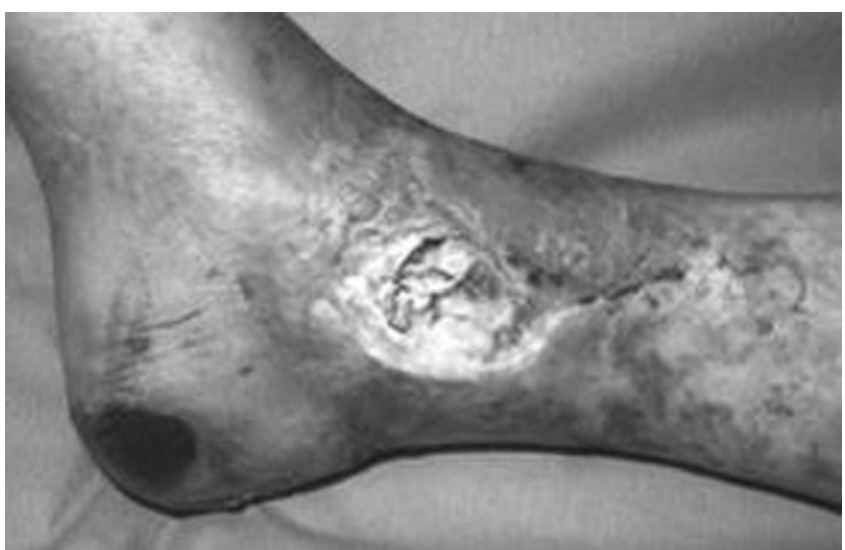

Abb. $2 \Delta$ Klinisches Bild eines chronischen Empyems des oberen Sprunggelenks mit begleitender Osteitis des Pilon tibiale

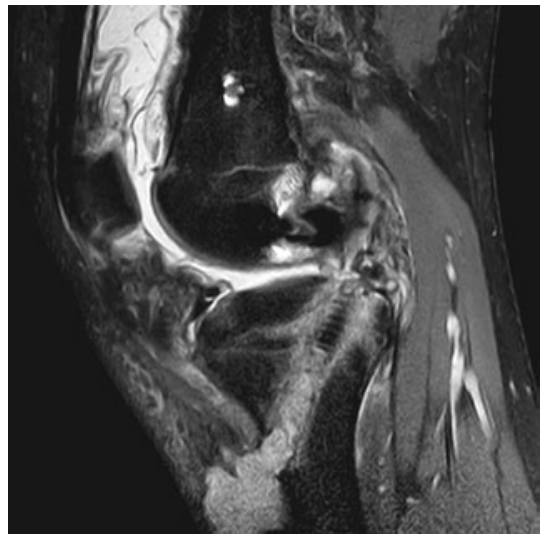

Abb. $3 \Delta$ MRT bei chronischem Kniegelenkempyem mit Infekt des tibialen Bohrkanals nach HKB-Ersatzplastik, MRT Magnetresonanztomografie, $H K B$ hinteres Kreuzband

geschehen auszugehen. Sollte im Punktat der Erregernachweis gelingen, wäre die Diagnose der Gelenkinfektion gestellt [21].

Als bildgebende Verfahren zur Diagnostik eines Kniegelenkinfekts sind die Röntgennativuntersuchung, die Sonografie und die Magnetresonanztomografie (MRT) zu nennen. Die konventionelle Röntgenaufnahme zeigt im Frühinfekt keinen Hinweis auf entzündliche Veränderungen mit Ausnahme von Lufteinschlüssen bei perforierenden Verletzungen oder einer Infektion mit Gas bildenden Keimen. Bei der chronischen Gelenkinfektion bzw. dem Spätinfekt finden sich Veränderungen wie Sequestrierung und Aufhellungen unterhalb des Knorpels oder auch eine Zerstörung der Gelenkflächen [4].
Magnetresonanztomografisch lassen meist ein Ödem von angrenzendem Knochen und Knorpel, gelegentlich Arrosionen am Knochen und Knorpel und der in der überwiegenden Zahl der Fälle bereits klinisch bekannte Erguss detektieren [4, 15]. Die Synoviahypertrophie ist im MRT gut zu diagnostizieren ( $\bullet$ Abb. 3 ).

Mittels Sonografie $[4,14,15]$ können der klinisch schon bekannte Gelenkerguss, aber auch eine Synoviaverdickung nachgewiesen werden.

Die CT-Untersuchung (CT: Computertomografie) sowie die Dreiphasenskelettszintigrafie haben in der Diagnostik einer Gelenkinfektion eine nachgeordnete Bedeutung $[4,15]$.

Zusammenfassend lässt sich sagen, dass für die Diagnosestellung einer Gelenkinfektion die Anamnese, die klinischen Symptome, Laboruntersuchungen, die Gelenkpunktion und die Sonografie bzw. das MRT entscheidend sind. Die Nativröntgenuntersuchung ist zur Diagnose einer akuten Gelenkinfektion nicht geeignet.

\section{Therapie}

Ziel der Behandlung von Gelenkinfektionen sind die dauerhafte Infektberuhigung sowie der Erhalt eines stabilen Gelenks und einer belastungsfähigen Extremität. Die Mobilität des Patienten soll möglichst wiederhergestellt und die berufliche und soziale Wiedereingliederung sollen ermöglicht werden.

Die akute und die chronische Infektion erfordern ein unterschiedliches Vor- gehen. Aufgrund der oben erläuterten pathophysiologischen Veränderungen ist von einer akuten Infektion innerhalb der ersten 7 Tage nach erstmaliger Manifestation der klinischen Symptome zu sprechen, bei länger andauernder Symptomatik ist von einer chronischen Gelenkinfektion auszugehen [11].

Die Behandlung von akuten Gelenkinfektionen stellt einen unfallchirurgischen Notfall dar! Sie bedarf der sofortigen operativen Intervention durch einen hierin erfahrenen Operateur.

Die Diagnosestellung einer Gelenkinfektion ist zu erzwingen, die Behandlung hat zeitnah, radikal und mit Konzept zu erfolgen. Verzögerungen in der Diagnostik oder eine insuffiziente Therapie resultieren in einer ausbleibenden Infektberuhigung mit desaströsen Folgen für den $\mathrm{Pa}$ tienten.

Die Auswertung der Daten von $90 \mathrm{~Pa}-$ tienten, die mit Schultergelenkempyem bei uns behandelt wurden, ergab vor Übernahme eine Dauer der Behandlung von durchschnittlich 123 Tagen mit im Mittel 5,5 ( 1 bis >20) infektberuhigenden Voroperationen (ohne Primäroperation). Ähnliche Ergebnisse erbrachte die Analyse von 84 Patienten mit chronischem Kniegelenkempyem. Die durchschnittliche Dauer der Vorbehandlung vor Aufnahme bei uns betrug 90 Tage (11 bis 600 Tage) mit im Schnitt 4,5 (0 bis 12) Voroperationen.

Für den Behandlungserfolg ist das radikale chirurgische Vorgehen entscheidend. 
Die richtige Vorgehensweise hängt nicht nur davon $a b$, ob eine akute und chronische Infektion vorliegt, sondern auch von der Vorbehandlung und Infektausdehnung sowie dem Ausmaß der Gelenkschädigung.

Bei der stadienadaptierten Behandlung $[9,21,22]$ stehen das arthroskopische oder das offene Vorgehen zur Auswahl. Akute Empyeme werden arthroskopisch behandelt, chronische Empyeme werden in der Regel offen chirurgisch versorgt. Bereits offen vorbehandelte Empyeme werden offen weiterbehandelt, Gelenkinfektionen mit Einbezug des gelenknahen Knochens können nicht arthroskopisch behandelt werden, auch hier ist eine offene Vorgehensweise erforderlich.

Die arthroskopische Behandlung ist bei akuten Empyem und Empyemen Stadium I (Synovialishyperämie und Erguss) und II (Synovialishypertrophie, Eiteransammlung im Gelenk) nach Gächter [9] sinnvoll und indiziert. Die Arthrotomie zur Behandlung einer Gelenkinfektion ist bei chronischen Empyemen und bei Empyemen Stadium III (Synovialisschwamm und beginnender Knorpelschaden) und IV (sog. Synovialismalignität) nach Gächter [9] notwendig.

Die arthroskopische Behandlung bedarf einer sorgfältigen Inspektion des Gelenks. Ein radikales Débridement $[9,13$, $21]$ ist erforderlich, ebenso die Lavage mit mindestens 101 Flüssigkeit sowie die Einlage eines oder mehrerer Antibiotikumträger. Diese antibiotikumhaltigen Vliese können über den Arbeitstrokar in das Gelenk eingebracht werden. Bei Infektion nach VKB-Ersatzplastik (VKB: vorderes Kreuzband) sollte ein Erhaltungsversuch des Kreuzbandersatzes unternommen werden [3]. Bei der Behandlung von akuten Empyemen ist eine geplante SecondLook-Operation nach 48-72 h sinnvoll.

Bei fortgeschrittenen Gelenkinfektionen sollte, wenn die Behandlung arthroskopisch begonnen wurde, frühzeitig auf ein offenes Verfahren gewechselt werden. Denn bei der Arthroskopie besteht die Gefahr, dass das Problem unterschätzt wird, woraus eine nicht ausreichend radikale Behandlung der Gelenkinfektion resultiert.

Beim offen chirurgischen Vorgehen ist die bilaterale Arthrotomie indiziert (Knie-

Trauma Berufskrankh 2014 • 16[Suppl 4]:439-443 DOI 10.1007/s10039-014-2086-2

(c) Springer-Verlag Berlin Heidelberg 2014

\section{U.-J. Gerlach · C. Grimme · R. Schoop · M. Borree Gelenkinfekt - eine Entität für die spezielle septische Chirurgie}

\section{Zusammenfassung}

Hintergrund. Die Gelenkinfektion stellt eine schwerwiegende Komplikation dar und führt bei ausbleibender oder inadäquater Behandlung zur irreversiblen Gelenkzerstörung. Pathogenese. Ursächlich für eine Gelenkinfektion sind endogen-hämatogene und exogene Faktoren. Bei unserem Patientengut wurden die Empyeme fast ausschließlich durch iatrogene Maßnahmen (arthroskopische Operationen, Punktionen, intraartikuläre Infektionen) verursacht oder waren Folge gelenknaher Frakturen und penetrierender Verletzungen.

Diagnose. Die Diagnosestellung erfordert neben der Laboruntersuchung, Nativröntgenaufnahmen und einer MRT-Untersuchung (MRT: Magnetresonanztomografie) auch die Gelenkpunktion mit Direktabstrich. Die Leukozytenzahl [Grenzwert: 25.000/ $\mu$ l $\left.\left(2,5 \times 10^{10} / \mathrm{I}\right)\right]$ muss bestimmt und möglicherweise vorliegende Bakterien müssen mikroskopisch mittels Gram-Färbung identifiziert werden.
Therapie. Beim akuten Gelenkempyem handelt es sich um einen unfallchirurgischen Notfall, der sofort operiert werden muss, da es aufgrund der pathophysiologischen Vorgänge rasch zu irreversiblen Knorpelschäden kommt. Dabei wird arthroskopisch vorgegangen. Von einem chronischen Empyem ist auszugehen, wenn die klinischen Symptome länger als 7 Tage bestehen. Seine Behandlung erfolgt durch Arthrotomie, Synovektomie und Entfernung des einliegenden Fremdmaterials inklusive eines evtl. vorhandenen Kreuzbandersatzes. Postoperativ ist von Beginn an eine intensive Übungsbehandlung erforderlich, auch unter Einsatz von Schmerzkathetern. Die Verlegung in ein Zentrum für Septische Chirurgie ist sinnvoll.

\section{Schlüsselwörter}

Knocheninfektion · Gelenkpunktion . Empyem · Arthroskopische Behandlung · Arthrotomie

\section{Joint infections - an entity for special septic surgery}

\section{Abstract}

Background. Joint infections can cause severe complications that result in irreversible joint destruction when treated inadequately. Pathogenesis. Reasons for joint infections consist of endogenous hematological and exogenous factors. Patients acquire empyema almost solely through iatrogenic measures (e.g. arthroscopic operations, punctures and intra-articular infections) or as a result of near joint fractures and penetrating injuries. Diagnosis. To make a correct diagnose, a joint puncture with a direct swab test is necessary in addition to laboratory examinations, $x$-ray and magnetic resonance imaging (MRI) examinations. The leucocyte count must be determined (threshold value 25,000/ $\mu \mathrm{l})$ as well as a gram stain to microscopically identify possible bacteria present.

Therapy. Acute joint infections are considered to be orthopedic emergencies necessi- tating immediate surgical treatment as irreversible cartilage damage can rapidly result due to the pathophysiological process. Acute joint empyema should be treated arthroscopically. Clinical symptoms lasting more than 7 days emanate in chronic empyema and should be treated by arthrotomy, synovialectomy and removal of extraneous material including a possible cruciate ligament replacement. Postoperatively, an immediate intensive physiotherapy is necessary, even under the use of pain catheters. The transfer to a centre for septic surgery is recommended.

\section{Keywords}

Infection - Joint puncture - Empyema .

Arthroscopic surgery $\cdot$ Arthrotomy gelenk, Ellenbogengelenk, oberes Sprunggelenk). Am Schultergelenk ist zumindest bei chronischen Infektionen neben dem ventralen der dorsale Zugang erforderlich, um radikal debridieren zu können. Ein Hüftgelenkempyem sollte nicht arthros- kopisch, sondern stets offen chirurgisch über einen Zugang behandelt werden.

Bei der offenen chirurgischen Behandlung ist die radikale Synovektomie (- Abb. 4) erforderlich. Begleitende Infektionen von gelenknahem Knochen müssen durch radikale Sequestrektomie 


\section{Septische Problemfälle}

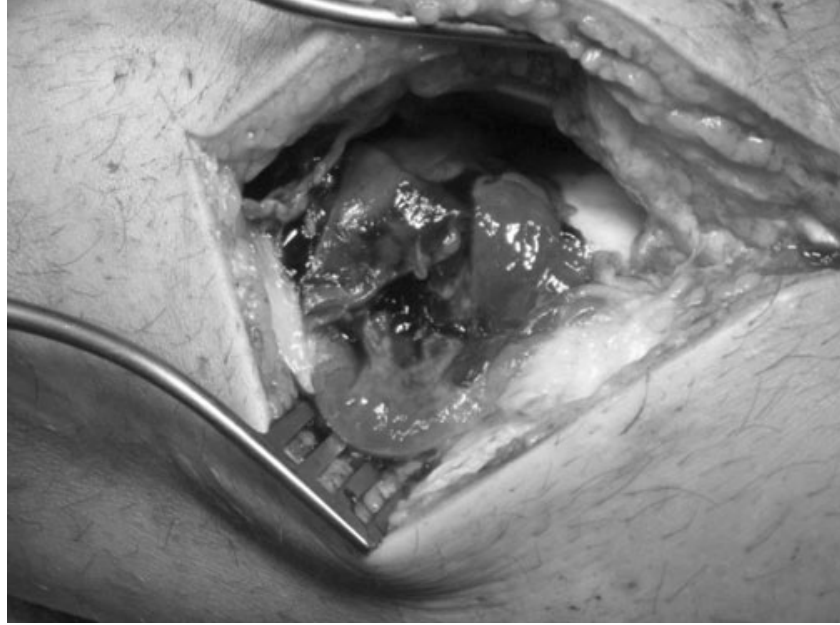

Abb. $4<$ Hypertrophe Synovia bei chronischem Kniegelenkempyem

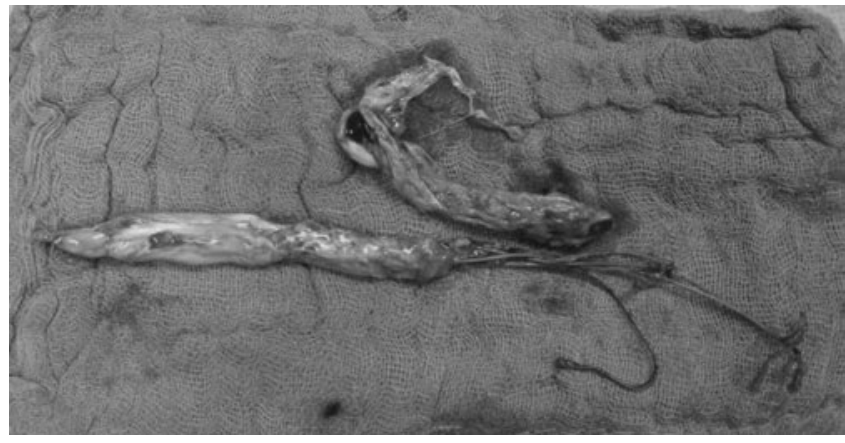

Abb. $5<$ Entfernter infizierter VKB-Ersatz, VKB vorderes Kreuzband

in gleicher Sitzung mit behandelt werden $[18,23]$. Implantate und Kreuzbandersatz müssen entfernt werden (• Abb. 5). Nach radikalem Débridement erfolgt die Einlage lokaler resorbierbarer Antibiotikumträger, und das Gelenk wird verschlossen. Selbstverständlich wird entnommenes Gewebematerial zur bakteriologischen und histologischen Untersuchung verschickt. Postoperativ ist eine kurzfristige systemische Antibiose (7 bis 10 Tage) erforderlich.

\section{Nachbehandlung}

Die postoperative Physiotherapie ist entscheidend und unerlässlich [22]. Zunächst unter ausreichender Schmerzmedikation, ggf. auch unter Schmerzkatheterbehandlung, erfolgen die Umlagerung auf einer Kirschner-Schiene, später die Behandlung mit der Motorschiene und die aktive Übungsbehandlung. Parallel zum operativen Vorgehen ist ein intensives Rehabilitationsprogramm unter krankengymnastischer Anleitung, hydrophysikalischer, sport- und ergotherapeutischer Behandlung erforderlich. Hilfsmittelversorgung und ggf. die Einleitung berufshelferischer Maßnahmen können im Verlauf notwendig werden. Die begonnene Physiotherapie sollte unbedingt auch nach der Entlassung aus der stationären Behandlung fortgeführt werden.

\section{Verlegung}

Gelenkinfektionen werden nicht selten zu spät erkannt und nicht suffizient chirurgisch behandelt. Konsequenz hieraus sind lange Krankheitsverläufe mit vielen frustranen Voroperationen und ein schlechteres funktionelles Ergebnis. Das Heilverfahren und der Zeitraum der Arbeitsunfähigkeit dauern länger an, es resultiert in der Regel eine höhere MdE (Minderung der Erwerbsfähigkeit). Deswegen ist die frühzeitige Verlegung in ein Septisches Zentrum sinnvoll.

\section{Fazit für die Praxis}

Bei Verdacht auf Gelenkinfektionen ist eine Diagnose zu erzwingen.
- Eine akute Gelenkinfektion stellt einen unfallchirurgischen Notfall dar.

- Die Behandlung hat stadienadaptiert zu erfolgen, entweder arthroskopisch oder offen chirurgisch.

- Bei fortgeschrittenen Gelenkinfektionen sollte, wenn arthroskopisch begonnen wurde, frühzeitig auf ein offenes Verfahren gewechselt werden.

- Bei chronischen oder voroperierten Empyem muss offen vorgegangen werden.

- Für den Behandlungserfolg ist das radikale chirurgische Vorgehen entscheidend.

- Die Einlage lokaler Antibiotikumträger und die kurzzeitige systemische Antibiose sind als unterstützende Maßnahmen anzusehen und entbinden nicht von einer radikalen chirurgischen Behandlung.

- Bei weitgehender Gelenkzerstörung und oder persistierender Instabilität ist die Arthrodese indiziert.

- Postoperativ ist der sofortige Beginn einer intensiven Übungsbehandlung für das funktionelle Ergebnis unerlässlich.

- Die frühzeitige Verlegung in ein Septisches Zentrum ist sinnvoll.

\section{Korrespondenzadresse}

Dr. U.-J. Gerlach

Abteilung für Septische Unfallchirurgie und Orthopädie, Berufsgenossenschaftliches Unfallkrankenhaus Hamburg,

Bergedorfer Straße 10, 21033 Hamburg

U.J.Gerlach@buk-hamburg.de

\section{Einhaltung ethischer Richtlinien}

Interessenkonflikt. U.-J. Gerlach, C. Grimme, R.

Schoop und M. Borree geben an, dass kein Interessenkonflikt besteht.

Dieser Beitrag beinhaltet keine Studien an Menschen oder Tieren.

The supplement containing this article is not sponsored by industry.

\section{Literatur}

1. Anders G (1984) Gelenkpunktionen und intraartikuläre Injektionen in ambulanten orthopädischen Einrichtungen. Beitr Orthop Traumatol 31:419-425 
2. Armstrong RW, Bolding F, Joseph R (1992) Septic arthritis following arthroscopy: clinical syndromes and analysis of risk factors. Arthroscopy 8:213-223

3. Attmanspacher W, Dittrich V, Stedtfeld W (1997) Behandlungsstrategie beim Kniegelenkinfekt nach vorderer Kreuzbandplastik. Arthroskopie 10:318322

4. Braunschweig $R$, Bergert $H$, Kluge $R$, Tiemann $A H$ (2011) Bildgebende Diagnostik bei Osteitis/Osteomyelitis und Gelenkinfekten. Z Orthop Unfall 149:436-448

5. Chapman MW (2001) Chapman's orthopaedics, 3. Aufl. Lippincott, Philadelphia, S 1800

6. D'Angelo GL, Ogilvie-Harris DJ (1988) Septic arthritis following arthroscopy, with cost/benefit analysis of antibiotic prophylaxis. Arthroscopy 4:10-14

7. Diefenbeck M, Abitzsch D, Hofmann GO (2012) Die Gelenkinfektion. Bekanntes und neue Trends. Unfallchirurg 115:489-495

8. Dittrich V, Attmanspacher W, Stedtfeld HW (2002) Gelenkinfektionen. Chirurg 73:959-967

9. Gächter A (1994) Gelenkinfekt: Arthroskopische Spülbehandlung - Hints und Tricks. Arthroskopie 7:98-101

10. Gray RG, Gottlieb NL (1983) Intra-articular corticosteroids. An updated assessment. Clin Orthop Relat Res 177:235-263

11. Heppert V, Glatzel U, Wagner C, Wentzensen A (2002) Komplikationsmanagement bei infizierten Kniegelenken. Trauma Berufskrankh 4:63-71

12. Holland C, Jaeger L, Smentkowski U et al (2012) Septische und aseptische Komplikationen in Verbindung mit Kortikoid-Injektionen: Eine Auswertung von 278 einschlägigen Fällen der Gutachterkommissionen und Schlichtungsstellen der Jahre 2005-2009. Dtsch Arztebl Int 109:425-430. DOI 10.3238/arztebl.2012.0425

13. Jerosch J (2004) Akuter Gelenkinfekt. Orthopade 33:1309-1320

14. Jerosch J, Prymka M (1998) Arthroskopische Therapie der septischen Arthritis - Operative Technik und Ergebnisse. Unfallchirurg 101:454-460

15. Linke R, Weidemann H, Militz M (2009) Bildgebende Diagnostik der Osteitis. Szintigraphie, PET oder MRT? Trauma Berufskrankh [Suppl 2] 11:193-202

16. Pioro MH, Mandell PF (1997) Septic arthritis. Rheum Dis Clin North Am 23:239-258

17. Riel KA, Primbs J, Bernett P (1994) Arthroskopische Überlaufsspüldrainage bei akuter postoperativer Kniegelenksinfektion - Langzeitergebnisse. Chirurg 65:1023-1027

18. Schmidt HGK, Gerlach UJ, Hadler D, Wurm M (2003) Therapie des posttraumatischen Ellengelenkempyems. Trauma Berufskrankh 5:55-64

19. Simank HG, Wadi B, Bernd L (2004) Gelenkempyeme. Orthopade 33:327-331

20. Smith NC (1987) Complications in arthroscopic surgery performed by experienced arthroscopists. Arthroscopy 4:215-221

21. Stutz G, Gächter A (2001) Diagnostik und stadiengerechte Therapie von Gelenkinfekten. Unfallchirurg 104:682-686

22. Stutz G, Kuster MS, Kleinstuck F, Gächter A (2000) Arthroscopic management of septic arthritis: stages of infections and results. Knee Surg Sports Traumatol Arthrosc 8:270-274

23. Walenkamp GH, Kleijn LL, Leeuw M de (1998) Osteomyelitis treated with gentamicin - PMMA beads. Acta Orthop Scand 69:518-522 